\title{
Treatment with Psychotropic Drugs in Oncology: Relevance Attributed to Possible Side Effects
}

\author{
Poças $\mathrm{A}^{1 *}$, Albuquerque $\mathrm{E}^{2}$ and Mónico $\mathrm{L}^{3}$ \\ ${ }^{1}$ Psychiatry Trainee, Leiria Hospital, Portugal \\ ${ }^{2}$ Consultant Psychiatry, Portuguese Oncology Institute of Coimbra, Portugal \\ ${ }^{3}$ Psychologist, $\mathrm{PhD}$ in Social Psychology, Coimbra University, Portugal
}

*Corresponding author: Ana Poças, Psychiatry Trainee, Leiria Hospital, Olhalvas St, Pousos, 2410-197 Leiria, Portugal, Tel: 912482690; E-mail: analuisapocas@gmail.com

\section{Research Article}

Volume 2 Issue 1

Received Date: February 02, 2018

Published Date: April 04, 2018

DOI: $10.23880 / \mathrm{mhrij}-16000115$

\section{Abstract}

Oncology patients are exposed to stress events associated with the diagnosis and treatment of cancer that can lead to significant losses in their quality of life. These patients may have significant psychiatric comorbidities and most of the times they are treated with chemotherapy which may have neuropsychiatric side effects or interact with psychotropic drugs. Patient's opinion and the shared therapeutic decision are the core of contemporary medicine. To know the preferences of the patients it is necessary to question them about this subject. We apply questionnaires to 83 oncology patients with mental health problems asking about the relevance they attributed to possible side effects of treatment with psychotropic. Regarding gender, there were no statistically significant differences in the relevance attributed to possible side effects of psychotropic drugs, except in the greater importance attributed by men to possible changes in sexual life and drug interactions. Those with higher educational levels attributed greater importance to some of the possible side effects of psychotropic drugs; as such schooling was positively associated with the relevance attributed to sleepiness, changes in sexual life and altered consciousness. We found that patients with long-term pathologies (Psychosis or Sleep Disorders) tend to attribute greater importance to a possible weight gain than patients with Adjustment Disorders. In patients who were still on antineoplastic treatment we verified that greater relevance was attributed to possible drug interactions. The different relevance attributed to the possible side effects of psychotropic drugs emphasizes the importance of paying attention to the specificities of each patient allowing the clinicians a more adequate therapeutic option.

Keywords: Psychotropic Drugs; Oncology; Mental Health; Chemotherapy 


\section{Mental Health \& Human Resilience International Journal}

\section{Introduction}

Oncology patients are exposed to stress events associated with the diagnosis and treatment of cancer that can lead to significant losses in their quality of life, implying the need for psychosocial adaptation of patients and their families. These patients may have significant psychiatric comorbidities and studies indicate that cancer patients are at an increased risk of developing Depressive Disorders [1]. These patients most of the times are treated with chemotherapy which may have neuropsychiatric side effects and some antineoplastic drugs may interact with psychotropic drugs. In addition, radiotherapy directed to brain regions may lead to the development of focal necrosis or leukoencephalopathy [1$3]$.

Patient's opinion and the shared therapeutic decision are the core of contemporary medicine [4]. Studies have shown that patients believe that psychotropic drugs have significantly more serious side effects compared, for example, to medications for heart disease [5].

Clinicians should be aware that their priorities may differ from those of their patients. To know the preferences of the patients it is necessary to question them about this subject. Literature is scarce on this topic, especially in this particular group of patients. For this reason, we have proposed to analyze the relevance attributed by cancer patients to possible side effects of treatment with psychotropic drugs.

\section{Methodology}

This is a prospective, observational and cross-sectional study in a sample of cancer patients followed in a Psychiatry consultation at the Portuguese Oncology Institute of Coimbra in treatment with psychotropic drugs.

Patient questionnaires were administered during prescheduled psychiatric consultations. It was collected sociodemographic data as well as clinical features. Patients were also asked about the relevance attributed to possible side effects of psychotropic drugs.

\section{Sample Description}

The sample consists of a total of 83 patients: 60 are women and 23 are men. The mean age is 57 years for women and 63 years for men surveyed. As far as marital status is concerned, 50 were married or living together; 7 were single; 15 divorced and 11 widowed. The majority of patients had a schooling equivalent to the 9th grade or less. 35 patients had studied up to the 4th year at the most; 23 to the 9th grade, 14 to the 12 th grade, and 11 had taken an upper course.

Regarding the distribution of patients due to cancer, we found that:

- 6 had the diagnosis of Head and Neck neoplasia;

- 14 of Gastro-intestinal neoplasia,

- 39 of Breast neoplasm;

- 2 of Gynecological neoplasia;

- 8 of Urological neoplasia;

- 6 of Lung neoplasm;

- 1 of Hepatic neoplasm;

- 2 of Blood neoplasia;

- 5 with another type of neoplasia.

- Regarding the psychiatric diagnosis, we verified that:

- 10 patients had Dysthymia;

- 37 had Depressive Disorders;

- 18 had Anxious Disorders;

- 8 had Adjustment reactions with Anxious / Depressive symptomatology;

- 2 had Bipolar Affective Disorders;

- 5 had Psychoses;

- 6 had Sleep Disorders.

\section{Results}

Regarding gender, there were no statistically significant differences in the relevance attributed to possible side effects of psychotropic drugs, except in the greater importance attributed by men to possible changes in sexual life ( $\mathrm{t}(81)=-3.497, \mathrm{p}<0.01)$ and drug interactions $\mathrm{t}(81)=-2.408, \mathrm{p}<0.05)$.

We observed that as the age increases, the weightrelated worry decreases $(r=0.395, p<0.01)$ as well as the concern with gastrointestinal changes $(r=0.219, \mathrm{p}=$ $0.047)$.

It was found that divorced individuals attributed greater importance to possible drug interactions comparing to single people ( 2.96 points more, $\mathrm{p}=0.04$ ) and to a possible weight gain in relation to married couples (4.23 points more, $p<0.01)$. Globally, married patients attributed less relevance to possible side effects of psychotropic drugs.

Those with higher educational levels attributed greater importance to some of the possible side effects of psychotropic drugs; as such schooling was positively associated with the relevance attributed to sleepiness 


\section{Mental Health \& Human Resilience International Journal}

( $r$ ho $=0.407, \mathrm{p}<0.01$ ), changes in sexual life (rho $=0.341$, $\mathrm{p}<0.02$ ) and altered consciousness ( $\mathrm{rho}=0.283, \mathrm{p}=0.09$ ).

It was found that patients with Bipolar Affective Disorder $(n=2)$ were the ones who attributed less relevance to possible side effects. Psychiatric diagnosis did not influence the relevance attributed except for weight gain where the mean attributed was higher in patients with Sleep or Psychopathology and lower in Adjustment Disorders with depressive and anxious symptomatology.

The organ/tissue affected by the neoplasia did not significantly influence the relevance attributed except in patients with Gastrointestinal neoplasia. These patients attributed greater importance to a possible weight gain. In patients with neoplasia in remission it was attributed greater relevance to drowsiness, $t(77)=4.1, p<0.01$. In the patients who were still in treatment it was attributed greater relevance to possible drug interactions $\mathrm{t}(81)=$ 2.23, $\mathrm{p}=0.028$.

\section{Discussion and Conclusion}

There are differences in the relevance attributed to possible side effects of psychoactive drugs according to sociodemographic characteristics such as age, sex, marital status, schooling; psychiatric and oncological diagnosis.

We found that patients with long-term pathologies (Psychosis or Sleep Disorders) tend to attribute greater importance to a possible weight gain than patients with Adjustment Disorders. These patients often have been on psychoactive drugs for several years and have had previous experience of taking drugs that have contributed to their weight gain. This prior experience may have an influence on their current perspective.

In patients who were still on antineoplastic treatment we verified that greater relevance was attributed to possible drug interactions. Patients undergoing antineoplastic drugs have often to follow prolonged treatments with medication whose side effects are very unpleasant and serious. This experience, or the fear that it may occur, may be responsible for the greater relevance attributed by them to possible drug interactions with psychotropic drugs.

The different relevance attributed to the possible side effects of psychotropic drugs emphasizes the importance of paying attention to the specificities of each patient allowing the clinicians a more adequate therapeutic option. Knowledge about possible side effects, fears and treatment duration certainly influence the relevance attributed by patients $[6,7]$.

In addition, the therapeutic relationship, the recognition of patient concerns and a clear explanation of the possible side effects of the drugs will be important elements in the perception and consequent relevance attributed by the patients [8].

\section{References}

1. Levenson JL (2006) Psychiatric Issues in Oncology. Primary Psychiatry.

2. Zabora J, BrintzenhofeSzoc K, Curbow B, Hooker C, Piantadosi S (2001) The prevalence of psychological distress by cancer site. Psychooncology 10(1): 19-28.

3. Weiss T, Nelson C, Tew W, Hardt M, Mohile SG, et al. (2015) The relationship among age, anxiety, and depression in older adults with cancer. Psychooncology 24(6): 712-717.

4. Eiring, Landmark BF, Aas E, Salkeld G, Nylenna M, et al. (2015) What matters to patients? A systematic review of preferences for medication-associated outcomes in mental disorders. BMJ Open 5(4).

5. Benkert Otto, Mechthild GM, Andreas H, Jürgen S, Simone EC, et al. (1997) Public Opinion on Psychotropic Drugs: An Analysis of the Factors Influencing Acceptance or Rejection. Journal of Nervous \& Mental Disease 185(3): 151-158.

6. Fann JR, Fan MY, Unutzer J (2009) Improving primary care for older adults with cancer and depression. J Gen Intern Med 417-424.

7. IOM (2001) Crossing the Quality Chasm: a new health system for the $21^{\text {st }}$ century. The Institute of Medicine, Committee on Quality of Health Care in America, USA.

8. IAPO (2004) What is Patient-Centred Healthcare? A review of Definitions and Principles. International Alliance of Patients' Organizations.

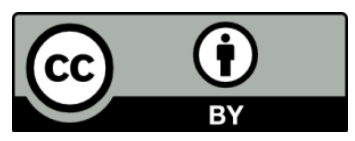

